\title{
Constant-net-time headway as key mechanism behind pedestrian flow dynamics
}

\author{
Anders Johansson* \\ ETH Zurich, \\ UNO C 11 Universitätstrasse 41, \\ 8092 Zurich, Switzerland
}

(Dated: November 5, 2018)

\begin{abstract}
We show that keeping a constant lower limit on the net-time headway is the key mechanism behind the dynamics of pedestrian streams. There is a large variety in flow and speed as functions of density for empirical data of pedestrian streams, obtained from studies in different countries. The net-time headway however, stays approximately constant over all these different data sets.

By using this fact, we demonstrate how the underlying dynamics of pedestrian crowds, naturally follows from local interactions. This means that there is no need to come up with an arbitrary fit function (with arbitrary fit parameters) as has traditionally been done.

Further, by using not only the average density values, but the variance as well, we show how the recently reported stop-and-go waves [Helbing et al., Physical Review E, 75, 046109] emerge when local density variations take values exceeding a certain maximum global (average) density, which makes pedestrians stop.
\end{abstract}

PACS numbers: 89.40.-a, 87.23.Ge, 12.00 .00

\section{INTRODUCTION}

With an increasing population and with more cost effective transportation, mass gatherings become more frequent. The total size of such gatherings are often as large as millions of people, for example during the inauguration ceremony of president Obama [1] and the Hajj pilgrimage to Mecca [2].

To guarantee the safety of the participants during such large mass gatherings, careful planning needs to be carried out by the organizer. During the last decades, numerous empirical studies [2, 3, 4, 5, 6, 7, 8, 9, 10, 11, 12, 13. have been performed on pedestrian crowds in different countries, in order to understand the dynamics of these crowds. Even though an understanding of crowd dynamics is a prerequisite for being able to plan a mass gathering, there is still no concensus on some of the most basic relations, such as how the flow of people (people per meter per second) depends on the crowd density (people per $\mathrm{m}^{2}$ ). Misconceptions of these basic relations may result in serious safety risks during mass gatherings [13].

Let us now start from the bottom up, and show how local interactions lead to certain flow-density relationships for the stream of pedestrians. Since movement and avoidance patterns of pedestrians tend to be rather complex, the traditional way to reduce complexity is to find a relation of the flow $Q\left(m^{-1} s^{-1}\right)$ as a function of the average density $\varrho\left(\mathrm{m}^{-2}\right)$. Using this relation, called the fundamental diagram, has been successful to some extent, but unfortunately there are large variations of these relations, among empirical studies carried out in different

*Electronic address: anders.johansson@gess.ethz.ch countries. All these studies agree on that walking speed of pedestrians is a decreasing function of density, but they disagree on how this function looks like. We will now demonstrate how the net-time headway, as a result of finite reaction times, is the key mechanism which can explain the discrepancies between data sets from different studies.

\section{REACTION TIME}

It is known from traffic science that finite reaction times are needed to explain instabilities in traffic flows [14. For pedestrian-flow dynamics, the role of finite reaction times has not been investigated in detail. By doing so, it turns out that the finite reaction time gives rise to a certain net-time headway, which is needed as a safety headway, to prevent accidental physical encounters with surrounding pedestrians.

Many-particle simulations [15, 16 coupled with empirical pedestrian-trajectory data [17 reveal the probabilitydensity function of delay times $T^{d}$ from a walking experiment [18] where two pedestrian streams are intersecting at a $90^{\circ}$ angle. The resulting distribution of delay times are shown in Fig. 1 .

Interestingly, the probability-density function of the delay curve is bi-modal. The first peak occurs at lower times than the typical response times, to visual or acoustic cues [19, 20]. Therefore, this peak must correspond to anticipated movements of the surrounding pedestrians. The second peak at around $0.45 \mathrm{~s}$ occurs at times which are significantly larger than the previously mentioned response times, but also lower than response times involving conscious reactions [14, 21]. Therefore, we conclude that this second peak corresponds to an unconscious re- 


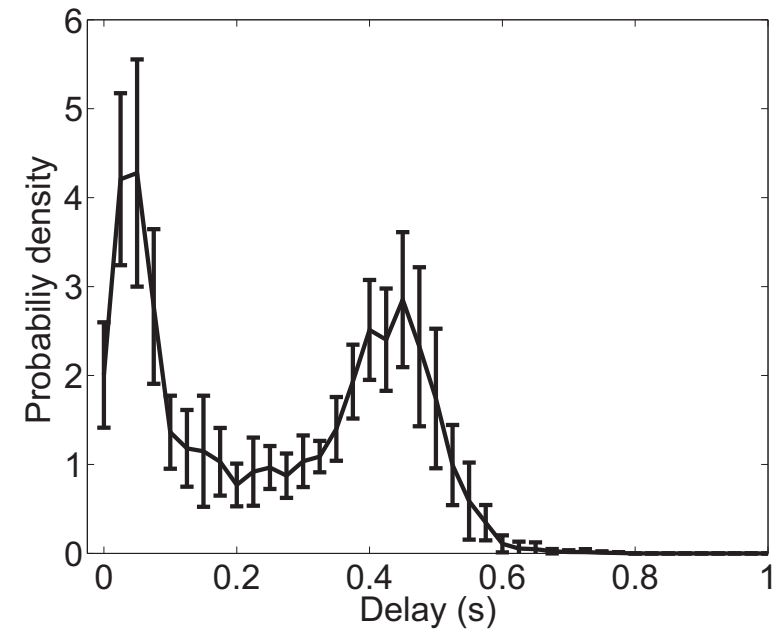

Figure 1: Probability-density function of the delay $T^{d}$. The error bars correspond to one standard deviation. Interestingly, the probability-density function is bi-modal. When the surrounding pedestrians are acting in a way that is easy to predict, extrapolation allows to anticipate their behaviors, while a delayed reaction results in cases of unexpected behaviors.

sponse, which is more complex than a simple reaction. In fact, it has been shown that reactions where there are more than one possible response (choice reaction time) as well as reactions to more complex cues (recognition reaction time) take significantly longer time [22].

We interpret the bi-modality as follows: When the surrounding pedestrians act in a way that is easy to predict, extrapolation allows to anticipate their behaviors, while a delayed reaction results in cases of unexpected behaviors.

\section{MODEL}

There has been a rich amount of microscopic models of pedestrian dynamics, for example the social-force model [15, 16] and cellular-automata models [23, 24, 25]. These models are able to reproduce various self-organization phenomena, such as lane and stripe formation [26], freezing by heating [27], Mexican waves in excitable media [28, intermittent outflows [29], stop-and-go waves and crowd turbulence [15].

When measuring empirical pedestrian flows and densities and then fitting a suitable curve to the data, one obtains a function which is useful for engineering involved in planning of pedestrian facilities. This pragmatic fit curve, however, does not provide any insight into the mechanisms and dynamics behind the pedestrian interactions and behaviours, leading to the aggregated data.

However, when plotting the fundamental diagrams obtained in various empirical studies (Fig. 3 (top)), one can see that each of the curves has a similar parabola-like

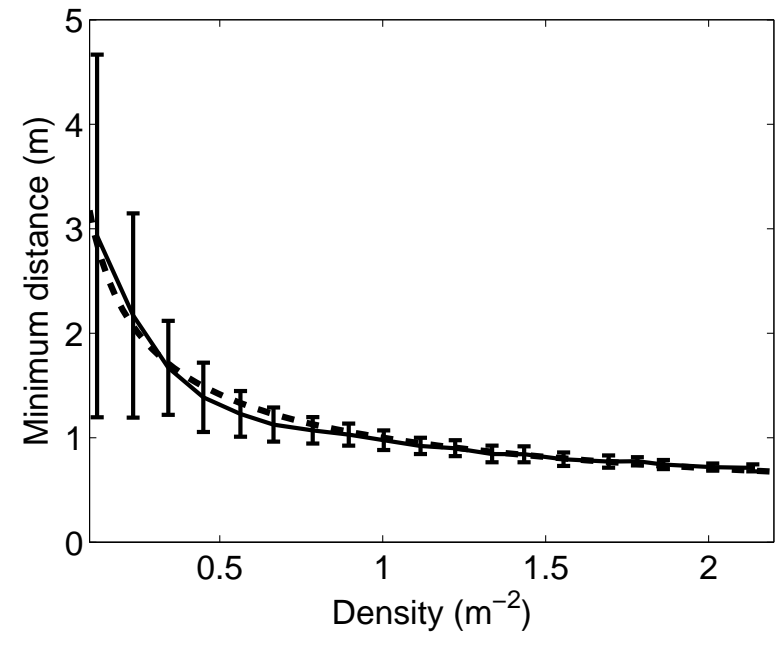

Figure 2: The distance between an arbitrary pedestrian $\alpha$ and the closest surrounding pedestrian $\beta$, as a function of global (average) crowd density $\varrho$. The solid line shows the average value \pm one standard deviation as error bars, and the dashed line shows the fit curve $1 / \sqrt{(} \varrho)$. The data is from Ref. [18]

shape. Nevertheless, the curves are quite different from one measurement site to another. One question remains to be answered: What, if any, is the common underlying principle of these curves?

In an attempt to bridge this knowledge gap, let us come back to the issue of reaction times, mentioned before. Since pedestrians have a typical reaction time $T^{d}=0.45$ $\mathrm{s}$ to unexpected behaviors of surrounding pedestrians, it would be natural that they compensate the risk of bumping into others, by keeping a certain safety time headway to the surrounding pedestrians 34 .

To connect the aggregated density to local interactions, let us approximate the mean distance between the centerof-masses of a pedestrian $\alpha$ and the closest pedestrian $\beta$ by $d=\left\langle d_{\alpha \beta}\right\rangle=1 / \sqrt{\varrho}$, where $\varrho$ is the global [35], (average) density. Note that this would hold only if the pedestrians were distributed into a square lattice, but for other density distributions, it will serve as a fair approximation (see Fig. 2).

The net distance is defined as $\hat{d}=d-2 r$, where $r=$ $1 /\left(2 \sqrt{\varrho_{\max }}\right)$ is the effective radius of a pedestrian, and $\varrho_{\max }$ is the largest measured density.

Assuming that the predecessor $\beta$ [36] would suddenly stop [29, it would take $\hat{T}=\hat{d} / v_{\alpha}=(1 / \sqrt{\varrho}-$ $\left.1 / \sqrt{\varrho_{\max }}\right) / v_{\alpha}$ seconds before a physical encounter with pedestrian $\alpha$ occurs, if $v_{\alpha}$ is the speed of pedestrian $\alpha$. We now show how the net-time headway $\hat{T}$ depends on the global density $\varrho$ by applying the above scheme to empirical data determined from different studies (see Fig. 3. bottom)).

Note that $\hat{T}$ saturates at a constant value, that is very similar to the response time to unexpected behaviors (see 
Fig. 11). However, in the data of Ref. [2] there is a transition at very high densities, where $\hat{T}$ suddenly increases. This can be interpreted in at least two ways:

- Hypothesis 1: When the density is very high, pedestrians start to have fear of crushing or asphyxia [30, and therefore want to increase the space around themselves (leading to higher net-time headways $\hat{T})$.

- Hypothesis 2: If the space in front of a pedestrian is too small (or the velocity is too low) it will no longer be possible to take normal steps. Rather, pedestrians will completely stop until they have gained enough space to make a step.

In previous work [30, Hypothesis 1 has been used. In this study, however, we will investigate Hypothesis 2. This interpretation would naturally explain the empirically observed stop-and-go waves analyzed in Ref. [2], and would further imply: Above an average density of 5 persons per $m^{2}$, the fundamental diagram will no longer describe the dynamics of the crowd well, since the flow rate is then alternating between movement and standstill rather than continuous.

In an attempt to unify all fundamental diagrams in the same framework, the following scheme is proposed:

Each pedestrian $\alpha$ has a free speed $v_{\alpha}^{0}=v_{\max }$ (which is an upper speed limit, occurring when $\varrho \rightarrow 0$ ). Each pedestrian also has a lower limit $v_{\min }$ of the speed. For $v<v_{\text {min }}$, pedestrians can no longer make normal steps, and would rather stop completely. For simplicity, these values are assumed to be the same for all pedestrians. It has been reported in Ref. [31 that the free (unconstrained) headways are exponentially distributed, where the constrained headways on the other hand, are limited by a desired minimum headway. Therefore, we propose that the net-time headway $\hat{T}$ is the key control parameter for the fundamental diagram. That is, pedestrians will decrease their speeds, if necessary, to assure a constant lower limit of the net-time headway $\hat{T}$. The fundamental diagram can now be specified as:

$$
v(\varrho)=\frac{d-2 r}{\hat{T}}=\frac{1 / \sqrt{\varrho}-1 / \sqrt{\varrho \max }}{\hat{T}}
$$

and bounded by $\left[v_{\min }, v_{\max }\right]$

It has been shown in Ref. [32] that each average density $\varrho$ corresponds to a distribution of local densities $\rho$, and we therefore approximate the density distribution with a Gaussian distribution $\mathcal{N}(\varrho, \sqrt{\varrho / 3})$ with mean $\varrho$ and standard deviation $\sqrt{\varrho / 3}$ (see Fig. 4).

According to Hypothesis 2, defined above, we get an extra constraint, saying that pedestrians will stop walking if they are too close to other pedestrians, which happens for $\rho \geq \varrho_{\max }$ (physical interaction). They will then resume walking again when they have enough space $L$ for taking a step. Since one step (for low walking speed)
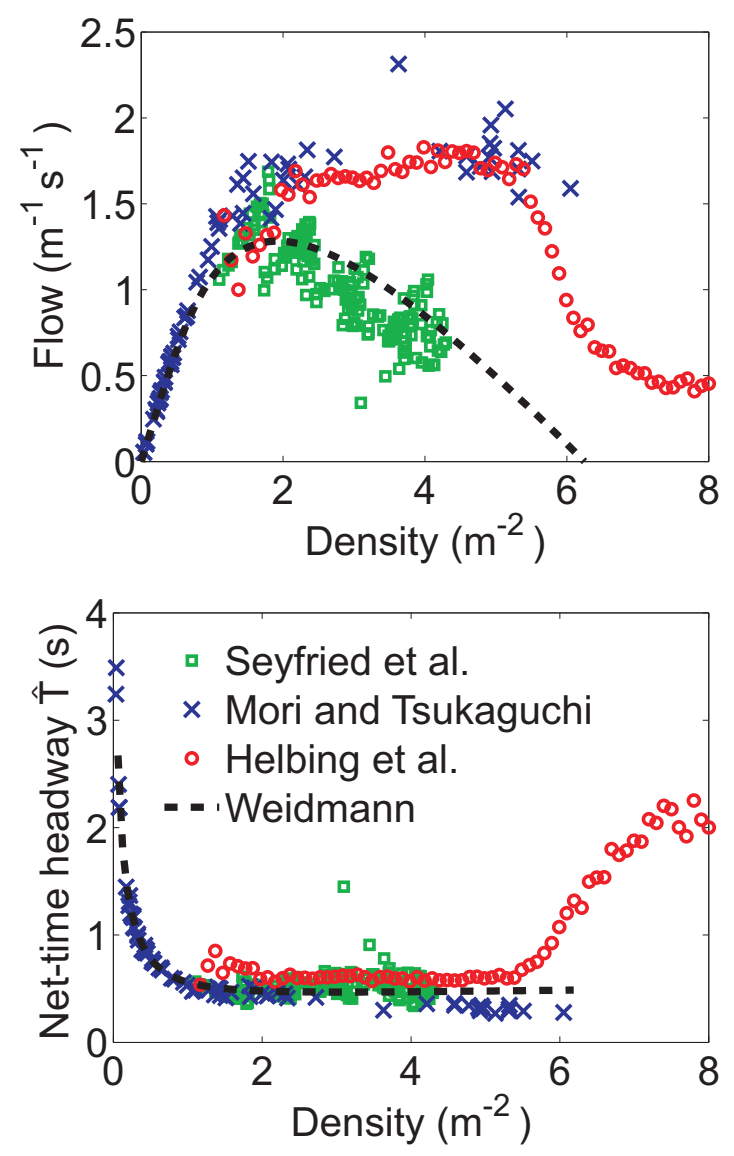

Figure 3: (Color online) Top: Flow as a function of density, for data from a number of empirical studies. Bottom: The net-time headways $\hat{T}$ as a function of density $\varrho$. $\hat{T}$ is most often bounded by a constant lower value of about $0.5 \mathrm{~s}$. In the data of Ref. [2], however, there is a transition for high densities where $\hat{T}$ suddenly increases. The data sets are the same as used in Ref. [2, i.e. the data from (Helbing et al.) correspond to local densities and flows.

needs approximately $L \approx 0.5 \mathrm{~m} \mathrm{[31}$, we get a new nettime headway $\hat{T}^{\prime}=L / v_{\min } \approx 10 \mathrm{~s}$, whenever $\rho \geq \varrho_{\max }$.

The fraction of pedestrians that are physically colliding with others, can be measured by integrating the probability-density-function of the Gaussian distribution (see Fig.6 (top)).

$$
f_{\text {stop }}=\int_{\varrho_{\max }}^{\infty} \mathcal{N}(\rho) d \rho
$$

with mean $\mu=\varrho$ and standard deviation $\sigma=\sqrt{\varrho / 3}$. Then, the mean net-time headway (see Fig. 6 (bottom)) is given by the fraction of stopped pedestrians as

$$
\langle\hat{T}\rangle=\left(1-f_{\text {stop }}\right) \hat{T}+f_{\text {stop }} \frac{L}{v_{\min }} .
$$

Figure 5 shows generated fundamental diagrams from Eq. 11 with the parameters $\hat{T}=0.5 \mathrm{~s}, \varrho_{\max }=5.4 \mathrm{~m}^{-2}$, 


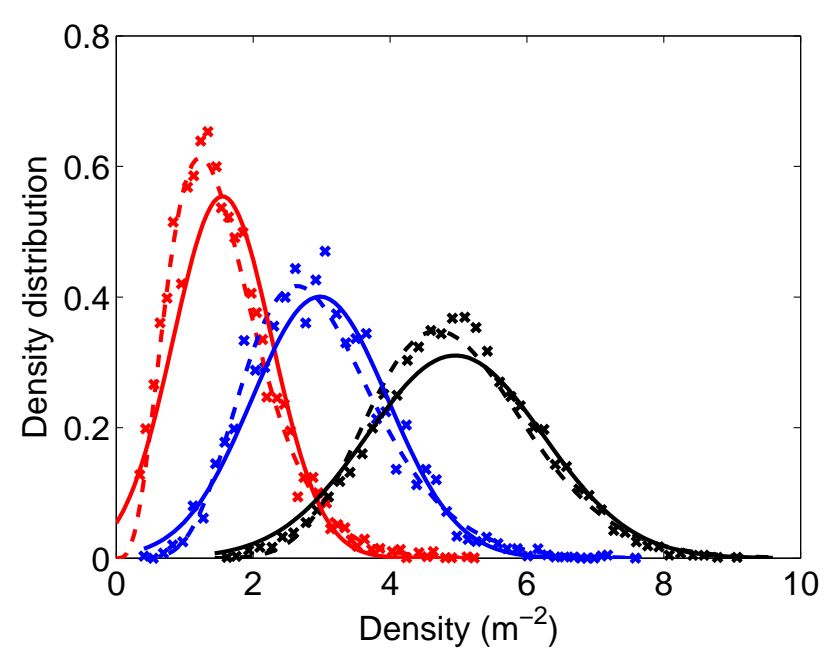

Figure 4: (Color online) Distributions of local densities $\rho$ for three different global (average) densities $\varrho=1.6,3$ and 5 pedestrians $/ \mathrm{m}^{2}$. The data comes from Ref. [2]. For each global density $\varrho$ a Beta distribution is fitted (dashed lines). However, Gaussian distributions (solid lines) also fit the data fairly well. The Gaussian distributions are produced with the parameters $\mu=\varrho$ and $\sigma=\sqrt{\varrho / 3}$.

and for different values of the free speed $v^{0}=v_{\max }$. Since $v^{0}$ only gives the upper limit of the velocity, fundamental diagrams with different $v^{0}$ converge at high enough crowd densities, given that all other parameters are fixed. The reason is that, for high density, the movement is transformed from individual walking to walking which is constrained by other pedestrians.

We now apply the method outlined above on different empirical fundamental diagrams. In all cases we use $\hat{T}=$ $0.5 \mathrm{~s}$ and $v_{\min }=0.06 \mathrm{~m} / \mathrm{s}$. Starting with Weidmann's [3] fundamental diagram, we have the parameters $\varrho_{\max }=$ $5.4 \mathrm{~m}^{-2}$ and $v^{0}=1.34 \mathrm{~m} / \mathrm{s}$, which is displayed in Fig. 7 (a, b) together with our curve, obtained by Eqs. (1) to (3).

Next, we apply our method to the fundamental diagrams of Refs. [4, [2] and [11, and obtain the results presented in Fig. 7 (c-h). The fit functions match all four different empirical data sets well. All parameters are kept constant over the different data sets, except the maximum density and the free speed, but these two values are obtained from the data, rather than tuned in order to fit the data.

\section{CONCLUSIONS}

The constant net-time headway is a natural safety mechanism to compensate for the reaction time to unexpected events. It has been demonstrated that various data sets, from different countries, all share the same net- time headway $\hat{T}=0.5 \mathrm{~s}$. The particular advantage of our
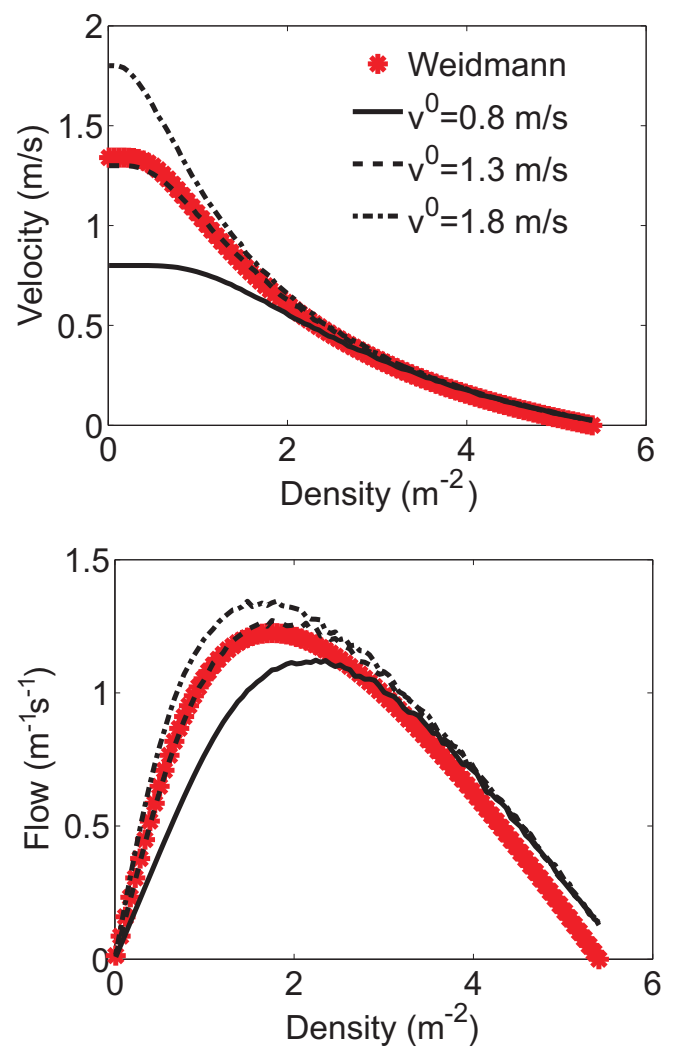

Figure 5: (Color online) Fundamental diagrams and velocitydensity relations generated by Eq. [1], for different free speeds $v^{0}$. Note how they all converge for large densities. As a comparison, the empirical fit curve by Weidmann [3] is shown.

method is that it follows naturally, without the need of an arbitrary fit function. Further, all the parameters are measurables, such as the free speed and the maximum density. There is not a single free parameter that must be tuned in order to fit the different data sets. However, it should be mentioned that even though the maximum density can be estimated, it can normally not be exactly determined from the data. This is addressed in recent work [33] that may make it possible to obtain culturally dependent parameters, such as the maximum density.

\section{ACKNOWLEDGMENTS}

The author is grateful for the partial financial support by the DFG grant He 2789/7-1.

He would also like to thank Dirk Helbing for his valuable comments, Serge Hoogendoorn for sharing data from his experiments, and to Habib Al-Abideen for sharing data from the Hajj. 

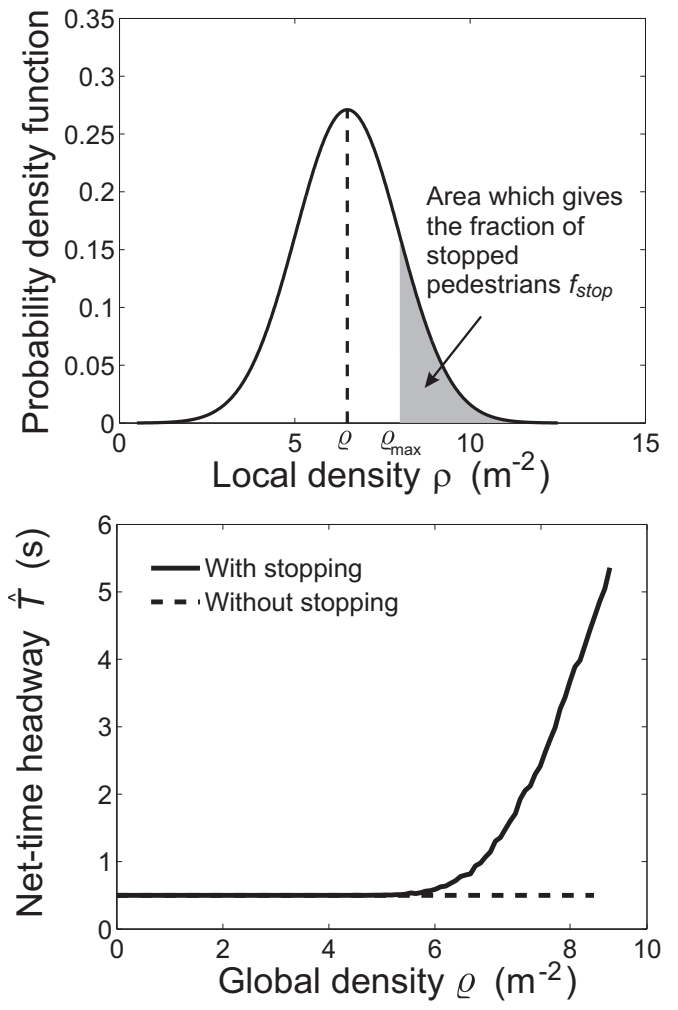

Figure 6: Top: The mean net-time headway $\langle\hat{T}\rangle$ is obtained via the fraction of pedestrians who are physically colliding with others, and is therefore stopping and temporarily increasing their net-time headway. This fraction is obtained by integrating over the probability-density-function of the local-density distribution, starting at local densities $\rho$ that are higher than the maximum global density $\varrho$. Bottom: The mean net-time-headway $\langle\hat{T}\rangle$ (solid line) as a function of the global density $\varrho$. The net-time headway without stopping is displayed as a dashed line.

[1] P. Taylor, How Officials Will Control the Crowds at Obama's Inauguration, Popular Mechanics, January 20, 2009.

[2] D. Helbing, A. Johansson, and H. Z, Al-Abideen, The Dynamics of Crowd Disasters: An Empirical Study, Phys. Rev. E 75, 046109 (2007).

[3] U. Weidmann, Transporttechnik der Fußgänger, ETHZürich, Schriftenreihe IVT-Berichte 90 (1993).

[4] M. Mori H. Tsukaguchi, A new method for evaluation of level of service in pedestrian facilities, Transportation Research A 21(3), pp. 223-234 (1987).

[5] J. J. Fruin, Designing for pedestrians: A level-of-service concept, Highway Research Record 355, 1-15 (1971).

[6] K. Ando, H. Ota, and T. Oki, Forecasting the flow of people, Railway Research Review 45, 8-14 (1988). (in Japanese).

[7] A. Polus, J. L. Schofer, and A. Ushpiz, Pedestrian flow and level of service, Journal of Transportation Engineer- ing 109, 46-56 (1983).

[8] R. A. Smith and J. F. Dickie (eds.), Engineering for Crowd Safety. (Elsevier, Amsterdam) (1993).

[9] K. Still, Crowd Dynamics, PhD Thesis (2000).

[10] K. Teknomo, Microscopic Pedestrian Flow Characteristics: Development of an Image Processing Data Collection and Simulation Model. PhD Thesis, Japan (2002).

[11] A. Seyfried, B. Steffen, W. Klingsch, and M. Boltes, The fundamental diagram of pedestrian movement revisited, J. Stat. Mech. P10002 (2005).

[12] T. Kretz, A. Grünebohm, and M. Schreckenberg, Experimental study of pedestrian flow through a bottleneck, J. Stat. Mech P10014 (2006).

[13] A. Schadschneider, W. Klingsch, H. Kluepfel, T. Kretz, C. Rogsch, and A. Seyfried, Evacuation Dynamics: Empirical Results, Modeling and Applications, Encyclopedia of Complexity and System Science, B. Meyers (Ed.) (Springer, Berlin, 2008). 

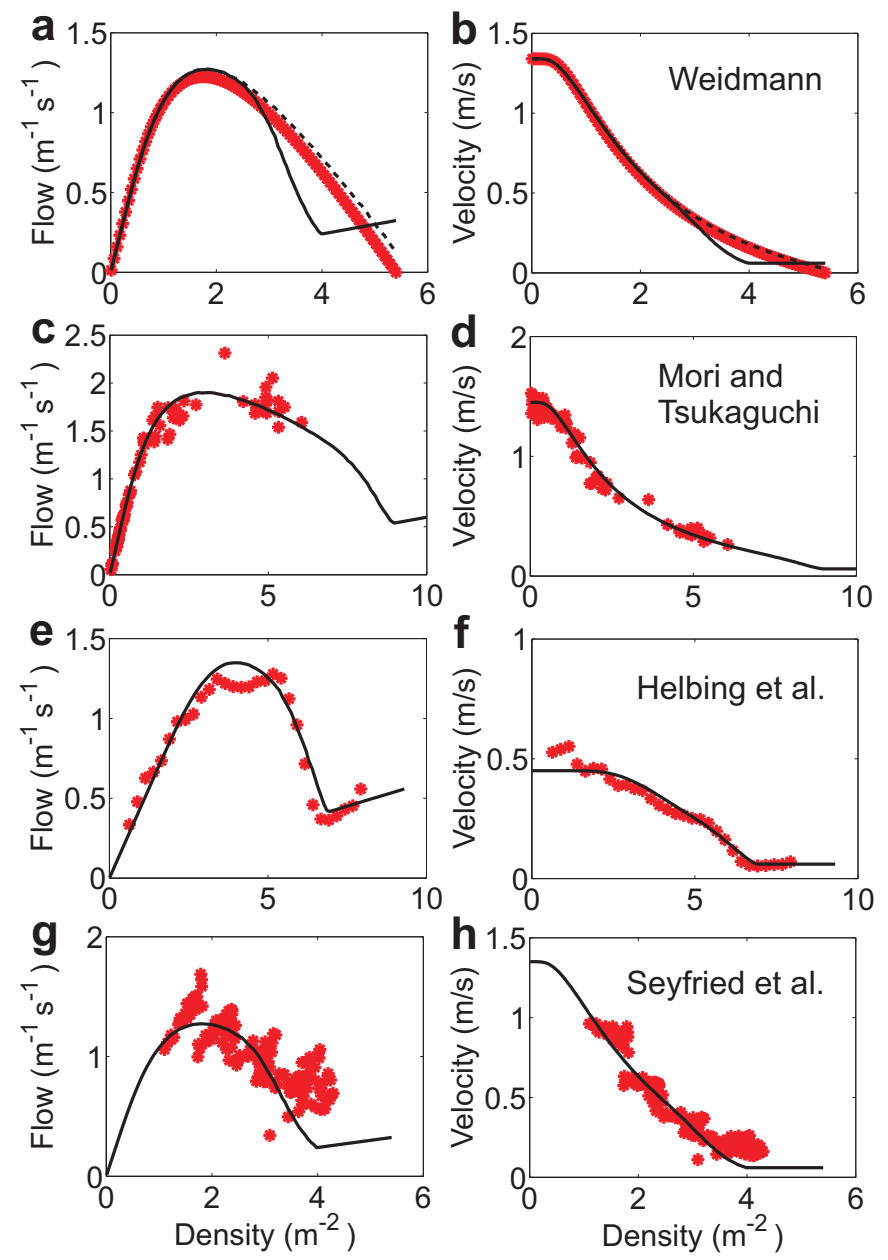

Figure 7: (Color online) Fundamental diagrams and velocitydensity relations generated by Eqs. (1) to (3), assuming a constant net-time headway $\hat{T}=0.5 \mathrm{~s}$ and a minimum velocity $v_{\text {min }}=0.06 \mathrm{~m} / \mathrm{s}$. Red markers represent empirical data and solid lines the theoretically expected relationships. a) Flow-density data, and b) velocity-density data by Weidmann [3], compared to a fundamental diagram generated with the parameters $\varrho_{\max }=5.4 \mathrm{~m}^{-2}$ and $v_{\max }=1.34 \mathrm{~m} / \mathrm{s}$. The dashed line shows the result when it is assumed that no pedestrians are stopping, i.e. $f_{\text {stop }}=0$. c) Flow-density data, and d) velocity-density data by Mori and Tsukaguchi [4], compared to a fundamental diagram generated with the parameters $\varrho_{\max }=12 \mathrm{~m}^{-2}$ and $v_{\max }=1.45 \mathrm{~m} / \mathrm{s}$. e) Flowdensity data, and f) velocity-density data from Helbing et al. [2], compared to a fundamental diagram generated with the parameters $\varrho_{\max }=9.3 \mathrm{~m}^{-2}$ and $v_{\max }=0.45 \mathrm{~m} / \mathrm{s} . \mathrm{g}$ ) Flow-density data, and h) velocity-density data from Seyfried et al. [11], compared to a fundamental diagram generated with the parameters $\varrho_{\max }=5.4 \mathrm{~m}^{-2}$ and $v_{\max }=1.35 \mathrm{~m} / \mathrm{s}$.

[14] M. Treiber, A. Kesting, and D. Helbing, Delays, inaccuracies and anticipation in microscopic traffic models, Physica A 360, pp. 71-88 (2006).

[15] D. Helbing and P. Molnár, Social force model for pedestrian dynamics, Phys, Rev. E 51, pp. 4282-4286 (1995).

[16] D. Helbing, I. Farkas, and T. Vicsek, Simulating dynam- ical features of escape panic, Nature 407, pp. 487-490 (2000).

[17] A. Johansson, Data-driven modeling of pedestrian crowds, PhD Thesis, TU Dresden (2009).

[18] S. P. Hoogendoorn, W. Daamen, and P. H. L. Bovy, Extracting microscopic pedestrian characteristics from video data, Annual Meeting Transportation Res. Board Pre-print CD-Rom, (Mira Digital Publishing, Washington, D.C) (2003).

[19] R. S. Woodworth and H. Schlosberg, Experimental Psychology. Henry Holt, New York (1954).

[20] A. T. Welford, Choice reaction time: Basic concepts. In A. T. Welford (Ed.), Reaction Times. Academic Press, New York, pp. 73-128 (1980).

[21] M. Green, How Long Does It Take to Stop? Methodological analysis of driver perception-brake times, Transport. Hum. Factors 2 pp. 195-216, (2000).

[22] F. C. Donders, On the speed of mental processes. Translated by W. G. Koster, 1969. Acta Psychologica, 30, pp. 412-431 (1868).

[23] A. Kirchner, K. Nishinari, and A. Schadschneider, Friction effects and clogging in a cellular automaton model for pedestrian dynamics, Phys. Rev. E 67, 056122 (2003).

[24] C. Burstedde, K. Klauck, A. Schadschneider, and J. Zittartz, Simulation of pedestrian dynamics using a twodimensional cellular automaton, Physica A, 295(3-4), pp. 507-525 (2001).

[25] W. G. Weng, T. Chen, H. Y. Yuan, and W. C. Fan, Cellular automaton simulation of pedestrian counter flow with different walk velocities, Phys. Rev. E 74, 036102 (2006)

[26] D. Helbing, L. Buzna, A. Johansson, T. Werner, Selforganized pedestrian crowd dynamics: Experiments, simulations, and design solutions, Transp. Sci. 39, pp. 1-24 (2005).

[27] D. Helbing, I. Farkas, and T. Vicsek, Freezing by heating in a driven mesoscopic system, Phys. Rev. Lett, 84, pp. 1240-1243 (2000).

[28] I. Farkas, D. Helbing, and T. Vicsek, Mexican waves in an excitable medium, Nature 419, pp. 131-132 (2002).

[29] D. Helbing, A. Johansson, J. Mathiesen, H. M. Jensen, and A. Hansen, Analytical approach to continuous and intermittent bottleneck flows, Phys. Rev. Lett. 97, 168001 (2006).

[30] W. Yu and A. Johansson, Modeling crowd turbulence by many-particle simulations, Phys. Rev. E 76, 046105 (2007).

[31] S. P. Hoogendoorn, W. Daamen, Pedestrian behavior at bottlenecks, Transp. Sci. 39(2), pp. 147-159 (2005).

[32] A. Johansson, D. Helbing, H. Z. A-Abideen, S. Al-Bosta, From crowd dynamics to crowd safety: A video-based analysis, Advances in Complex Systems 11(4), pp. 497527 (2008).

[33] U. Chattaraj, A. Seyfried, and P. Chakroborty, Comparison of pedestrian fundamental diagram across cultures, Advances in Complex Systems 12, 393 (2009).

[34] Note that we use the peak at longer delays since this peak corresponds to unexpected events. Even though there is a peak at lower delay times, there is no guarantee that the behaviours of others can always be anticipated, and therefore the maximum peak must be used as a safety time.

[35] The global density $\varrho$ is defined as the number of people within a certain area, divided by that area. The local den- 
sity $\rho[2$ on the other hand, is defined via a bell-shaped weight function, where the influence of close pedestrians is larger than the influence of remote pedestrians.
[36] This assumes a following behaviour for uni-directional streams, which is most often the case for empirical pedestrian studies. 